\title{
Physisorption of molecular hydrogen in curved carbon nanomaterials: a computational study
}

\author{
D. J. Durbin ${ }^{1,2}$, N. L. Allan ${ }^{2}$ \& C. Malardier-Jugroot ${ }^{1}$ \\ ${ }^{1}$ Department of Chemistry and Chemical Engineering, \\ Royal Military College of Canada, Canada \\ ${ }^{2}$ School of Chemistry, University of Bristol, UK
}

\begin{abstract}
Hydrogen physisorption on carbon nanomaterials is a promising method of hydrogen storage because carbon materials are cheap, abundant and light weight. However, storage is difficult because dispersion forces between $\mathrm{C}$ and $\mathrm{H}$ are weak. Curved carbon substrates are more promising than planar systems because the increased level of $\mathrm{sp}^{3}$-hybridization enhances $\mathrm{H}_{2}$ physisorption. The present study uses density functional theory to model large fullerenes, single-walled carbon nanotubes and graphene to investigate the interaction with $\mathrm{H}_{2}$; decorating platinum is also considered. We conclude that $\mathrm{H}_{2}$ can be stored in fullerenes without an energy input if the $\mathrm{H}_{2}$ molecules are more than $3 \AA$ from the carbon surface and more than $2 \AA$ from each other. In addition, confinement effects are observed when hydrogen is stored in fullerenes rather than nanotubes - storage in nanotubes is more favourable for systems with small diameters.

Keywords: hydrogen storage, curvature, confinement, fullerenes, carbon nanotubes, graphene, platinum, physisorption, fuel cells.
\end{abstract}

\section{Introduction}

Hydrogen gas has high potential as a fuel in fuel cells vehicles because of its high gravimetric energy density; unfortunately, it also has a very low volumetric density. Compact and efficient storage techniques are required. Traditional methods of compression and liquefaction are inadequate because of their high cost and inability to achieve sufficient compression. Alternative storage methods of metal and non-metal hydrides and absorption to carbon substrates are all currently of considerable current interest [1-4]. 
Hydrogen physisorption on carbon nanomaterials is one promising possibility. This storage technique has the advantage of using a cheap, light weight and abundant carbon nanomaterial, but it does not involve potentially awkward chemical reactions. Unfortunately, $\mathrm{C} \cdots \mathrm{H}_{2}$ dispersion forces are weak so the materials are often activated by the introduction of $\mathrm{sp}^{3}$-hybridized carbon or addition of metals, while ensuring that chemisorption is suppressed [5-7]. Even still, low temperatures and high pressures are required to achieve acceptable hydrogen storage capacities. Previous ab initio studies have found hydrogen gravimetric storage capacities as large as $19 \mathrm{wt} \%$ on packed carbon nanotubes (CNTs) at $77 \mathrm{~K}$ and $100 \mathrm{~atm}[8]$.

Curved rather than planar carbon materials are beneficial because a larger $\mathrm{sp}^{3}$ hybridized content in the structure makes hydrogen physisorption more favourable [7, 9]. Many studies have investigated such curved carbon surfaces. Most of these analyses were performed using only a small carbon surface with as little as five atoms [10-12]. Due to the small surface, these studies can miss the effects of multiple $\mathrm{C} \cdots \mathrm{H}_{2}$ interactions and they are vulnerable to edge effects.

To prevent this, the present study uses Density Functional Theory (DFT) to model entire $\mathrm{C}_{n}$ fullerenes where $n=20,60,180,540,960$. These fullerenes are compared to infinite $(m, m)$ single-walled CNTs (SWCNTs) where $m=3,5,9$ and to large graphene fragments to study the effects of confinement. Finally, the effects of exohedral and substitutional platinum and non-metal substitutional dopants (boron, nitrogen and oxygen) are considered in an effort to increase the favourability of $\mathrm{C} \cdots \mathrm{H}_{2}$ interactions. The results presented in this paper use the B3LYP functional and LANL2MB basis set. More detailed results incorporating dispersion effects with more extensive basis sets will be presented in a future paper by the authors.

\section{Computational method}

Calculations were performed using DFT as implemented in Gaussian 09 [13]. The hybrid B3LYP functional was used because of its reliability for modelling transition metals and it is not very computationally expensive $[14,15]$. The LANL2MB basis set was also employed because its use of previouslydetermined effective core potentials [16-18] greatly decreases computation time. The B3LYP functional and LANL2MB basis set have been shown to produce reliable results for systems containing similar carbon substrates [19, 20]. Still, they do not consider the important dispersion effects. To account for this, the B97D functional was used in more detailed studies; these results will be presented in a future work. Systems studied with B3LYP and with B97D show similar trends; B3LYP is, therefore, acceptable for the purposes of this paper.

Calculations were performed on carbon systems containing one $\mathrm{H}_{2}$ molecule, one exohedral $\mathrm{Pt}$ atom and one substitutional dopant atom where applicable. The carbon systems include entire $\mathrm{C}_{n}$ fullerenes $(n=20,60,180,540,960),(m, m)$ SWCNTs $(m=3,5,9)$ and graphene fragments. The nanotubes were represented as $8.5 \AA$ segments with 1D periodic boundary conditions; the graphene sheet measured 14.5 x $14.8 \AA$ and was hydrogen-terminated. This area is large enough 
that edge effects should be unimportant. Similar systems containing a large carbon surface with single dopants have been used successfully in previous modelling studies $[19,20]$.

Coordinates for the structures of the smaller fullerenes $(20 \leq n \leq 540)$ were obtained from the Computational Chemistry List, Ltd. on-line fullerene database compiled by Cramer at the University of Minnesota. Coordinates for the $C_{960}$ structure were provided by Henrard at the University of Namur. Coordinates for the SWCNTs were generated by TubeGen On-line (version 3.4) created by Frey and Doren at the University of Delaware. All systems were optimized without constraints.

\section{Results and discussion}

This study investigated the $\mathrm{C} \cdots \mathrm{H}_{2}$ interaction in a variety of $\mathrm{C}_{n}$ fullerenes $(n=$ $20,60,180,540,960$ ) (fig. 1) by analysing $\mathrm{C} \cdots \mathrm{H}_{2}$ and $\mathrm{H}_{2} \cdots \mathrm{H}_{2}$ bond distances and the fullerene- $\mathrm{H}_{2}$ interaction energy, $\Delta E$. This is given by eqn. (1) for systems containing only one $\mathrm{H}_{2}$ molecule and by eqn. (2) for systems with multiple $\mathrm{H}_{2}$.

$$
\begin{gathered}
\Delta E=E_{\text {fullerene } \mathrm{H}_{2}}-\left[E_{\text {fullerene }}+E_{\mathrm{H}_{2}}\right] \\
\Delta E=E_{\text {fullerene: } n \mathrm{H}_{2}}-\left[E_{\text {fullerene: }(n-1) \mathrm{H}_{2}}+E_{\mathrm{H}_{2}}\right]
\end{gathered}
$$

a)

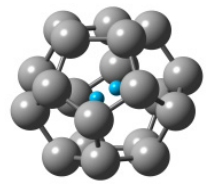

b)

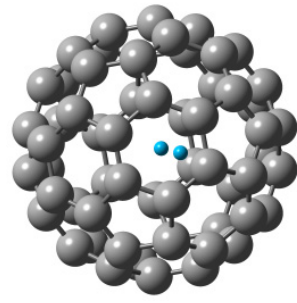

c)

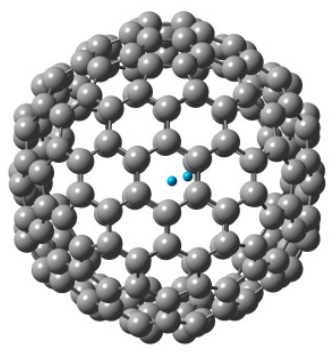

d)

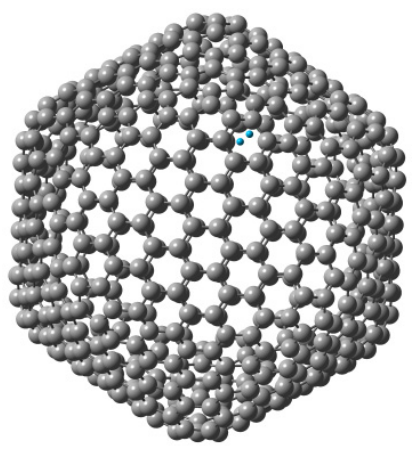

e)

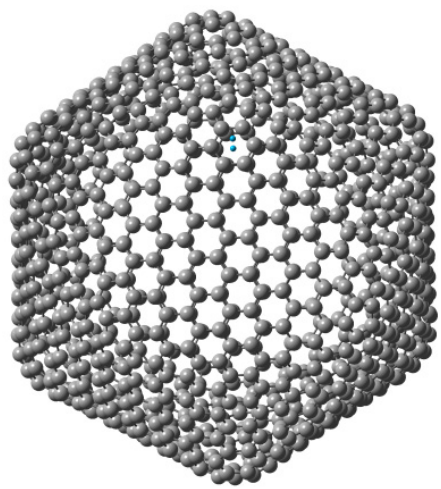

Figure 1: $\quad \mathrm{C}_{n}$ fullerenes optimized with one internal $\mathrm{H}_{2}$ molecule $(n=20,60$, 180,540 and 960 for $(\mathrm{a})-(\mathrm{e})$ respectively) $(\mathrm{C}=$ grey, $\mathrm{H}=$ blue $)$. 
Systems containing exohedral or substitution platinum (fig. 2) or no Pt were analysed with $\mathrm{H} 2$ stored inside the fullerene to determine the optimal $\mathrm{C} \cdots \mathrm{H} 2$ distance. C60 and C180 were further analysed to determine the possible effects of boron, nitrogen and oxygen substitutional dopants as well as multiple $(2-6)$ H2 molecules (section 3.1).

Although platinum was included in the systems, $\mathrm{Pt}-\mathrm{H}$ bonds are not formed because the initial position of $\mathrm{H}_{2}$ inside the fullerene presents a large geometric and energetic barriers to chemisorption.

To understand the systems better, the smaller fullerenes $(n=20,60,180)$ were compared with $(m, m)$ SWCNTs $(m=3,5,9)$ of similar diameter to determine the effect of confinement on hydrogen storage. The larger fullerenes, $\mathrm{C}_{540}$ and $\mathrm{C}_{960}$, were compared with graphene to determine if these fullerenes are so large that confinement becomes negligible to the point that graphene itself is a good model for the system; in addition, large fullerenes contain flat surfaces that can be compared directly to flat graphene (section 3.2).
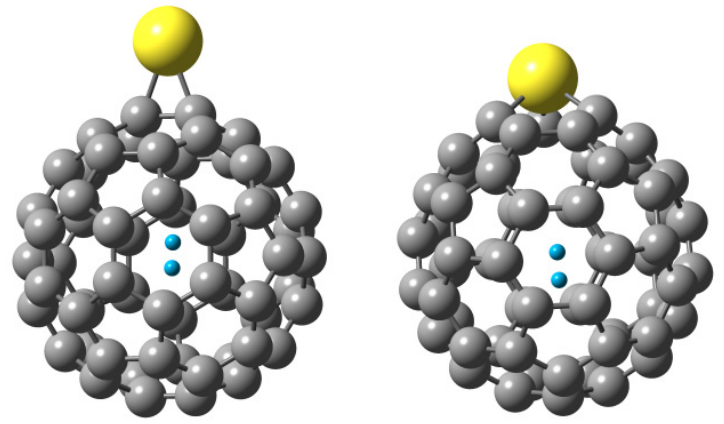

Figure 2: $\quad \mathrm{C}_{60}$ fullerenes optimized with one internal $\mathrm{H}_{2}$ molecule and exohedral (left) or substitutional (right) platinum $(\mathrm{Pt}=$ yellow, $\mathrm{C}=$ grey, $\mathrm{H}=$ blue).

\subsection{Optimal $\mathrm{C} \cdots \mathrm{H}_{2}$ distance}

The $\mathrm{C} \cdots \mathrm{H}_{2}$ distance is critical to determining the stability of a hydrogen storage system. This is observed, for example, with $\mathrm{C}_{20}$ fullerenes where confining even one $\mathrm{H}_{2}$ molecule is energetically unfavourable by $525 \mathrm{~kJ} / \mathrm{mol}$ with respect to the separate systems. This might be expected because the maximum possible $\mathrm{C} \cdots \mathrm{H}_{2}$ distance is $1.9 \AA$. This is as much as $1 \AA$ less than the combined van der Waals radii of carbon and hydrogen (1.7 and $1.2 \AA$ respectively) and so leads to unfavourable Pauli repulsion.

When $\mathrm{H}_{2}$ is placed inside larger fullerenes, the $\mathrm{C} \cdot \cdot \mathrm{H}_{2}$ distance can be greater than $2.9 \AA$ and so there are not significant repulsive interactions between carbon and hydrogen. The addition of further hydrogen to relatively small fullerenes such as $\mathrm{C}_{60}$, however, becomes unfavourable because of the $\mathrm{H}_{2} \cdots \mathrm{H}_{2}$ interactions. When there is only one $\mathrm{H}_{2}$ molecule in $\mathrm{C}_{60}$, the shortest $\mathrm{C} \cdots \mathrm{H}_{2}$ distance is $2.9 \AA$. When additional $\mathrm{H}_{2}$ molecules are added, the $\mathrm{C} \cdots \mathrm{H}_{2}$ distances decrease to as 
little as $2.3 \AA$ for $2-4 \mathrm{H}_{2}$ molecules and $2.2 \AA$ for $5-6 \mathrm{H}_{2}$. Correspondingly, there is a repulsive interaction of $105-131 \mathrm{~kJ} / \mathrm{mol}$ per $\mathrm{H}_{2}$ for $2-4 \mathrm{H}_{2}$ molecules and $184-236 \mathrm{~kJ} / \mathrm{mol}$ for $5-6 \mathrm{H}_{2}$ (fig. 3).

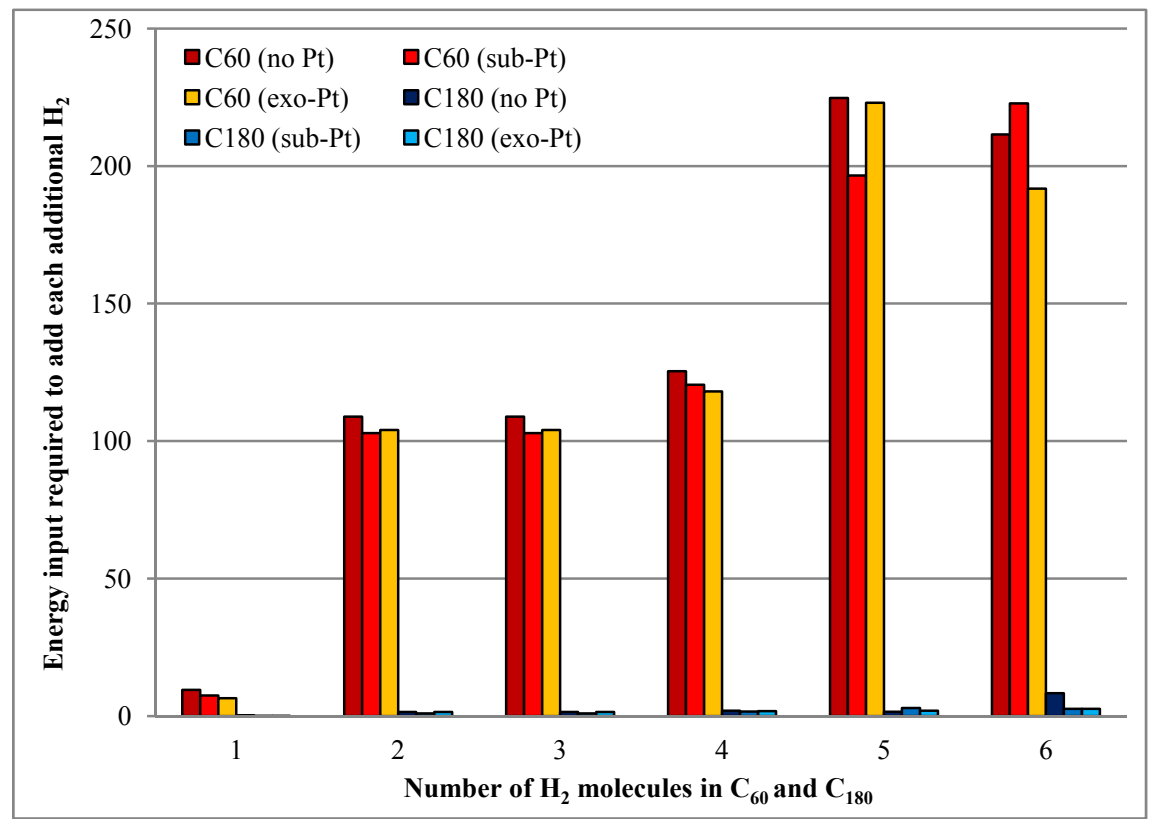

Figure 3: The energy input required to add additional $\mathrm{H}_{2}$ molecules to $\mathrm{C}_{60}$ containing no platinum or substitutional or exohedral platinum dopants for $1-6 \mathrm{H}_{2}$ molecules.

In all cases the $\mathrm{H}_{2}$ molecules orient in the centre of the fullerene to minimize their contact with the carbon surface and with each other (fig. 4). The molecules
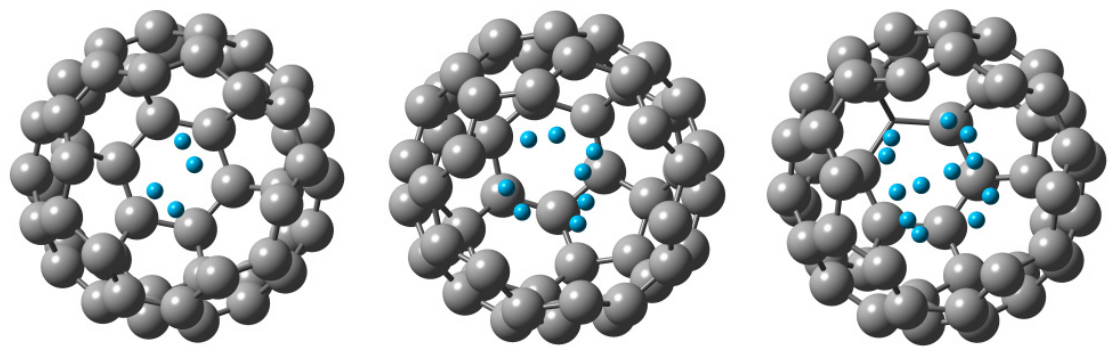

Figure 4: $\quad \mathrm{C}_{60}$ fullerenes optimized with two (left), four (middle) and six (right) internal $\mathrm{H}_{2}$ molecules. The topmost six carbon atoms have been excluded so that the $\mathrm{H}_{2}$ molecules can be observed more clearly $(\mathrm{C}=$ grey, $\mathrm{H}=$ blue $)$. 
cluster in the centre in an attempt to keep the $\mathrm{H}_{2} \cdots \mathrm{H}_{2}$ distances greater than $2 \AA$ and the $\mathrm{C} \cdots \mathrm{H}_{2}$ distances greater than $2.9 \AA$. When the distances become smaller than these optimal values, repulsive interactions dominate.

Similar central clustering of the $\mathrm{H}_{2}$ molecules is observed in $\mathrm{C}_{180}$ systems. However, up to six $\mathrm{H}_{2}$ molecules (inclusive) can be placed within the fullerene without causing significant unfavourable $\mathrm{C} \cdots \mathrm{H}_{2}$ or $\mathrm{H}_{2} \cdots \mathrm{H}_{2}$ interactions (fig. 3); this is consistent with the $\mathrm{C} \cdots \mathrm{H}_{2}$ distance remaining above $3 \AA$. This suggests that hydrogen can be stored in fullerenes if the system is large enough to allow the $\mathrm{H}_{2}$ molecules to remain at least $3 \AA$ from the carbon surface.

The additions of platinum and non-metal (boron, nitrogen, oxygen) dopants have a negligible effect on the $\mathrm{C} \cdots \mathrm{H}_{2}$ interactions. In previous studies of $\mathrm{H}_{2}$ and $\mathrm{CO}$ adsorbed on a platinum/carbon substrate, alterations to the substrate had a notable effect on the substrate-gas interaction $[19,20]$. The same effect is likely not observed here because in the previous study, the $\mathrm{H}_{2}$ molecules were able to easily interact with the platinum; in contrast, there are significant physical boundaries in the present study. In addition, the weak $\mathrm{H}_{2}$ interaction with the substrate is not influenced by the metal-carbon or dopant-carbon interactions as was observed in previous studies [21].

\subsection{Confinement effects}

The effects of $2 \mathrm{D}$ vs. 3D confinement on hydrogen storage within carbon nanomaterials were studied by comparing fullerenes with $(m, m)$ single-walled carbon nanotubes of similar diameters (fig. 5). $\mathrm{C}_{20}, \mathrm{C}_{60}$ and $\mathrm{C}_{180}$ were compared with $(3,3),(5,5)$ and $(9,9)$ SWCNTs respectively.

In addition, the larger fullerenes, $\mathrm{C}_{540}$ and $\mathrm{C}_{960}$, were compared with graphene. Because the shape of fullerenes changes from spherical to polyhedral (fig. 1) and the volume within the fullerene increases as the number of carbon atoms increases, flat graphene is often taken as a good approximation of these systems. If this is correct then curvature and confinement effects should be negligible in large fullerenes.
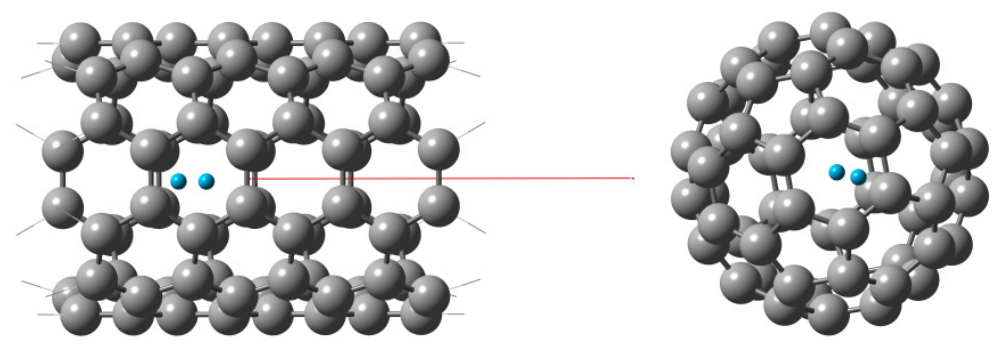

Figure 5: $\quad$ SWCNT $(5,5)$ (left) and $\mathrm{C}_{60}$ (right) optimized with one internal $\mathrm{H}_{2}$ molecule showing the comparable diameters of the systems $(\mathrm{C}=$ grey, $\mathrm{H}=$ blue, vector used for periodic boundary calculations $=$ red). 
Analysis of $\mathrm{H}_{2}$ in $\mathrm{C}_{20}$ and $\mathrm{SWCNT}(3,3)$ shows that it is unfavourable to store $\mathrm{H}_{2}$ within either small-diameter system; however, the interaction energy is lower in the nanotube. The energy required to put a $\mathrm{H}_{2}$ molecule into $\mathrm{C}_{20}$ (3D confinement) is over twice that required to place a $\mathrm{H}_{2}$ into $\operatorname{SWCNT}(3,3)$ (2D confinement) (499 kJ/mol for $\mathrm{C}_{20}$ vs. $236 \mathrm{~kJ} / \mathrm{mol}$ for $\left.\operatorname{SWCNT}(3,3)\right)$. This is likely to result from the larger number of $\mathrm{C} \cdots \mathrm{H}_{2}$ interactions in the fullerene.

As expected, larger systems that have negligible $\mathrm{C} \cdots \mathrm{H}_{2}$ interactions in the highly confined structure $\left(\mathrm{C}_{60}, \mathrm{C}_{180}\right)$ also show minimal interactions in their comparable nanotubes (SWCNT $(5,5)$, SWCNT $(9,9)$ respectively). When the larger polyhedron fullerenes, $\mathrm{C}_{540}$ and $\mathrm{C}_{960}$, are compared with a graphene segment, negligible $\mathrm{C} \cdots \mathrm{H}_{2}$ interactions are observed in all systems. Therefore, it appears that confinement and curvature are insignificant in such large fullerenes and so flat graphene is an acceptable approximation.

\section{Conclusion}

The present study analysed $\mathrm{H}_{2}$ stored within fullerenes and single-walled carbon nanotubes to understand $\mathrm{C} \cdots \mathrm{H}_{2}$ interactions in curved carbon nanomaterials. $A b$ initio calculations were used to model $\mathrm{C}_{n}$ fullerenes $(n=20,60,180,540$, 960) to determine the optimal $\mathrm{C} \cdots \mathrm{H}_{2}$ and $\mathrm{H}_{2} \cdots \mathrm{H}_{2}$ distances. Hydrogen storage in fullerenes was also compared to that in SWCNTs $(m, m)$ with similar diameters to determine the effects of confinement. Finally, the impact of exohedral and substitutional platinum as well as non-metal (boron, nitrogen, oxygen) substitutional dopants was also considered.

$\mathrm{C} \cdots \mathrm{H}_{2}$ interactions become negligible outside the combined van der Waals radii of carbon and hydrogen (1.7 and $1.2 \AA$ respectively); $\mathrm{H}_{2} \cdots \mathrm{H}_{2}$ interactions are unfavourable below $2 \AA$. Therefore, $\mathrm{H}_{2}$ can be stored in fullerenes without an energy input when these distances are respected. The addition of platinum (exohedral, substitutional) or non-metal (substitutional) dopants does not affect this. Endohedral platinum was not considered because $\mathrm{H}_{2}$ chemisorbed on $\mathrm{Pt}$ creates strong $\mathrm{Pt}-\mathrm{H}$ bonds; these would require a large energy input to break to release $\mathrm{H}_{2}$ and so are also undesirable in a storage material. The optimal $\mathrm{Pt}-\mathrm{H}$ interaction would be an intermediate between chemisorption and the physisorption analysed in this study.

Confinement effects are observed when spherical fullerenes are compared to SWCNTs. This is most prominent in smaller structures as can be observed with $\mathrm{H}_{2}$ stored in $\mathrm{C}_{20}$ versus SWCNT $(3,3)$; storage in the nanotube requires less than half of the energy input per $\mathrm{H}_{2}$ added. Confinement (and curvature) appears to be negligible in large fullerenes, such as $\mathrm{C}_{540}$ and $\mathrm{C}_{960}$. It is possible that the addition of metals dopants has a larger effect in systems with lower confinement. These results are still under investigation, as are studies with more extensive basis sets and explicit account taken of dispersion. 


\section{References}

[1] Demirel, Y., Energy production, conversion, storage, conservation and coupling: Springer, 2012.

[2] Khan, M.T.I., M. Monde, and T. Setoguchi, Hydrogen gas filling into an actual tank at high pressure and optimization of its thermal characteristics. Journal of Thermal Science, 2009. 18(3): p. 235-240.

[3] Jorgensen, S.W., Hydrogen storage tanks for vehicles: Recent progress and current status. Current Opinion in Solid State and Materials Science, 2011. 15(2): p. 39-43.

[4] Tozzini, V. and V. Pellegrini, Prospects for hydrogen storage in graphene. arXiv preprint:1207.5703, 2012.

[5] Yürüm, Y., A. Taralp, and T.N. Veziroglu, Storage of hydrogen in nanostructured carbon materials. International Journal of Hydrogen Energy, 2009. 34(9): p. 3784-3798.

[6] $\mathrm{Xu}, \mathrm{W} . \mathrm{C}$, et al., Investigation of hydrogen storage capacity of various carbon materials. International Journal of Hydrogen Energy, 2007. 32(13): p. 2504-2512.

[7] Tibbetts, G.G., G.P. Meisner, and C.H. Olk, Hydrogen storage capacity of carbon nanotubes, filaments, and vapor-grown fibers. Carbon, 2001. 39(15): p. 2291-2301.

[8] Assfour, B., et al., Packings of carbon nanotubes - new materials for hydrogen storage. Advanced Materials, 2011. 23(10): p. 1237-1241.

[9] Sofo, J.O., A.S. Chaudhari, and G.D. Barber, Graphane: A two-dimensional hydrocarbon. Physical Review B, 2007. 75(15): p. 153401.

[10] Elias, D.C., et al., Control of graphene's properties by reversible hydrogenation: evidence for graphane. Science, 2009. 323(5914): p. 610613.

[11] Tozzini, V. and V. Pellegrini, Reversible hydrogen storage by controlled buckling of graphene layers. Journal of Physical Chemistry C, 2011. 115(51): p. 25523-25528.

[12] Wang, L. and R.T. Yang, Hydrogen storage properties of carbons doped with ruthenium, platinum, and nickel nanoparticles. Journal of Physical Chemistry C, 2008. 112(32): p. 12486-12494.

[13] Frisch, M., et al., Gaussian 09, 2009, Gaussian Inc.: Wallingford, CT.

[14] Lee, C., W. Yang, and R.G. Parr, Development of the Colle-Salvetti correlation-energy formula into a functional of the electron density. Physical Review B, 1988. 37(2): p. 785.

[15] Chen, Z. and J. Yang, The B3LYP hybrid density functional study on solids. Frontiers of Physics in China, 2006. 1(3): p. 339-343.

[16] Hay, P.J. and W.R. Wadt, Ab initio effective core potentials for molecular calculations. Potentials for the transition metal atoms Sc to $\mathrm{Hg}$. Journal of Chemical Physics, 1985. 82(1).

[17] Hay, P.J. and W.R. Wadt, Ab initio effective core potentials for molecular calculations. Potentials for $K$ to Au including the outermost core orbitals. Journal of Chemical Physics, 1985. 82: p. 299. 
[18] Wadt, W.R. and P.J. Hay, Ab initio effective core potentials for molecular calculations. Potentials for main group elements $\mathrm{Na}$ to Bi. Journal of Chemical Physics, 1985. 82: p. 284.

[19] Durbin, D.J. and C. Malardier-Jugroot, Density functional theory analysis of metal/graphene systems as a filter membrane to prevent CO poisoning in hydrogen fuel cells. Journal of Physical Chemistry C, 2010. 115(3): p. 808815.

[20] Durbin, D.J. and C. Malardier-Jugroot, Theoretical investigation of the use of doped graphene as a membrane support for effective $C O$ removal in hydrogen fuel cells. Molecular Simulation, 2012. 38(13): p. 1061-1071.

[21] Groves, M.N., C. Malardier-Jugroot, and M. Jugroot, Improving platinum catalyst durability with a doped graphene support. Journal of Physical Chemistry C, 2012. 116(19): p. 10548-10556. 